\title{
THE PENN STATE SPECTROSCOPIC SURVEY TELESCOPE
}

L. W. Ramsey and D. W. Weedman

The Pennsylvania State University

525 Davey Laboratory

University Park, PA 16802

\section{ABSTRACT}

To map the distribution of galaxies and quasars in the universe, we desire a telescope large enough to obtain spectra to about 20 th magnitude in under 20 minutes integration and which is devoted entirely to such spectroscopic survey projects in dark time. We also desire extensive bright time observing to monitor large numbers of G-M stars with high-resolution spectroscopy for many years in search of solar-like activity. These projects are suited to a telescope with a large fixed primary, assembled from many spherically figured segments. We present a design for such a telescope that consists of 73 segments, each of $0.9-\mathrm{m}$ diameter and $26-\mathrm{m}$ radius of curvature. Mirror blanks of this size can be cut from standard Pyrex sheets. Effective aperture of the telescope exceeds 7-m; the focal plane system can track objects for 40 minutes, and sky coverage of 48 degrees is obtained by using a fixed tilt for the primary and making the entire telescope and dome rotatable. The focal plane system is lightweight and precisely pointable because spectrographs are coupled to the focus by fiber optic cables. Off-the-shelf components and existing technology are used to keep engineering and development costs low; we must remain within a budget feasible for a university. Because the telescope will be equipped with standard, minimal instrumentation and is intended for very routine observing programs, operating costs will also be low. Hardware components are now being assembled in a laboratory to develop the focal plane control system and the mirror support system.

Proceedings of the IAU Colloquium No. 79: "Very Large Telescopes, their Instrumentation and Programs", Garching, April 9-12, 1984. 


\section{INTRODUCTION}

Despite long standing interest, spectroscopic data for large numbers of objects in well defined samples have been difficult to obtain. Examples are many. The Markarian survey of galaxies began appearing in 1967 and eventually included 1500 galaxies, of which even now only 1000 have redshifts. With the resources of Palomar Observatory at their disposal, Schmidt and Green (1983) required several years to accumulate an all sky quasar sample to 16 th mag. Just to obtain 2400 redshifts of galaxies brighter than 14.5 magnitude required years of observing with many telescopes (Huchra et al. 1983). No systematic monitoring of spectral features in stars has yet compared to the Mt. Wilson efforts to follow changes in $\mathrm{H}$ and $\mathrm{K}$ emission in solar-like stars (Wilson 1978, Vaughn and Preston 1980). Note that none of the systematic studies mentioned have been done at National Observatories; virtually all astronomers recognize the importance of such systematic data, but the varied and topical demands on National facilities have generally squeezed out routine, long term programs. It is unlikely that this situation will change with the construction of large National telescopes. There is a desperate need for routine spectral data for relatively bright objects - galaxies and quasars to 19th mag. and high resolution spectra of stars to 12th mag. Such objects are just too bright for attention by expensive, 10-m class telescopes. Integration times would be short, but typically only one object could be observed per pointing (surface density of such objects being sufficiently low) so much observing time would be wasted simply in acquisition.

To satisfy the scientific need for spectroscopic surveys, we would like a telescope which is: a. routinely devoted to spectroscopic observing, b. large enough to obtain spectra to 20 th mag. with integration times of order 10 minutes, c. quickly moveable to new objects, d. cheap enough that long term devotion to routine programs is competitive with more glamorous projects. All of these needs are met by a telescope utilizing a fixed, spherical primary. We present a design for such a telescope whose construction we are proposing and for which fund-raising efforts have begun at The Pennsylvania State University.

As demonstrated by the success of the Arecibo Radio Telescope, a fixed spherical primary provides a relatively inexpensive way to obtain large collecting area. Several authors have suggested analogous designs for optical telescopes (e.g. Mertz 1980, Schectman 1983). The advantage for an optical telescope is that the primary can be built up of an array of small segments, each of which is easy to figure to a slow, spherical figure. There is no fundamental technological limitation to the size of the primary that can be constructed in this way, with individual segment size being determined by the cost drivers of mirror blank, figuring, and support. Determining the optimum 
combination of these is an important question for judging the cost, but not the feasibility, of an "Arecibo" optical telescope. The disadvantage of such a telescope that accounts for previous lack of interest among optical astronomers is the small field of view resulting from the correction for spherical aberration; this restricts the utility for wide field imaging. This is of no concern for a spectroscopic survey telescope; a field of $1^{\prime}$ is easily obtained. Similarly, constraints imposed by the needs of speckle interferometry or infrared astronomy do not apply so a phased array is unnecessary. This greatly simplifies segment alignment. We doubt that there will ever be a shortage of interesting objects that are known from imagery or non-optical surveys but whose optical spectroscopic characteristics need to be determined.

\section{TELESCOPE CONCEPT}

In order to obtain substantial sky coverage with a true Arecibo design, it is necessary to have a very fast primary whose total area is substantially

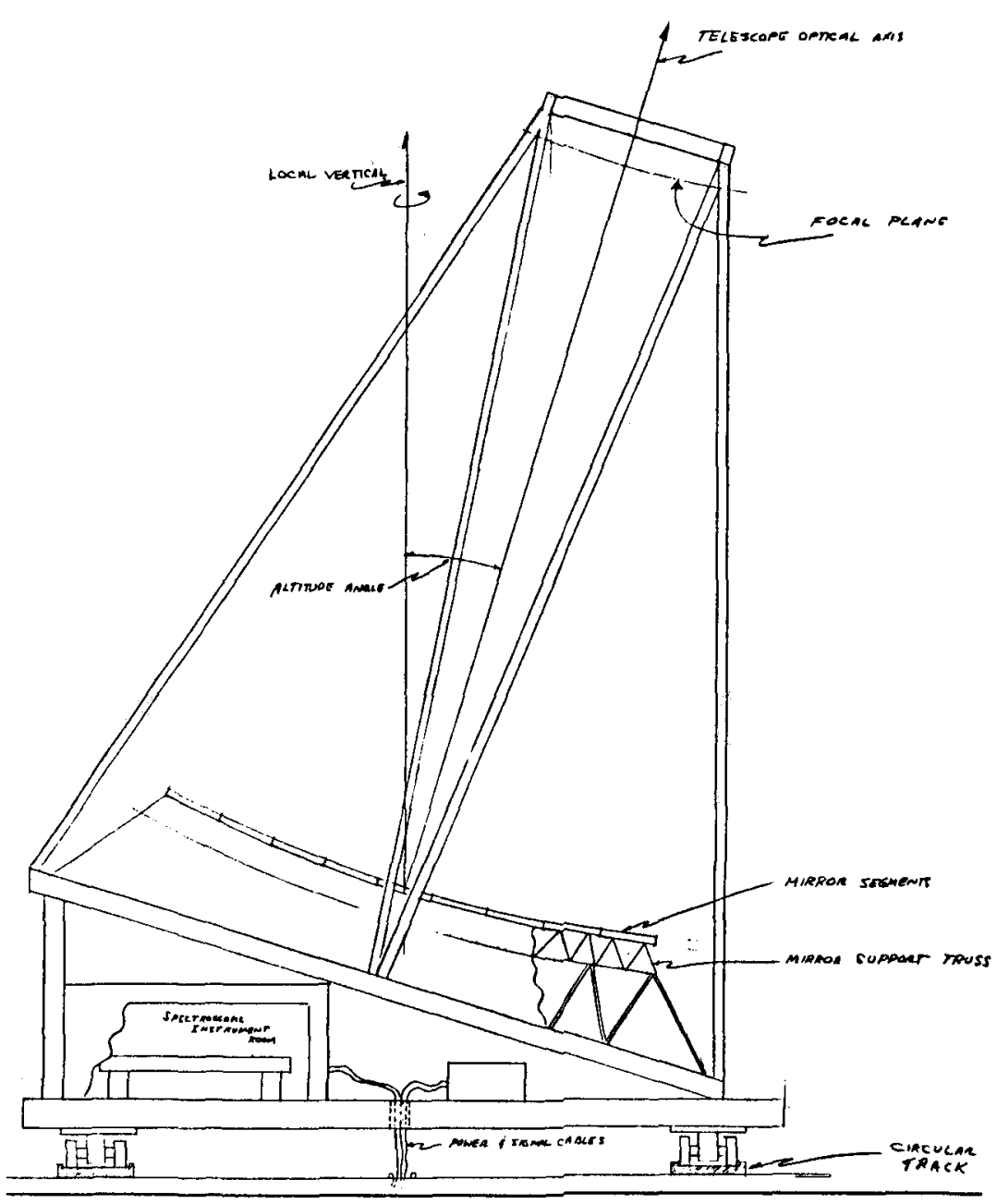
larger than the aperture illuminated for any single observation. Otherwise, the primary must be moveable, and that introduces the need for active alignment of the segments. We propose a hybrid design which utilizes segments, has large sky coverage, but for which the gravity vector of the primary never changes. This is accomplished by rotating the primary in azimuth without tilting it in altitude. This rotation is not for tracking but only for access to

Figure 1: Fixed-altitude telescope with spherical primary. 
different declination ranges. In the design illustrated (Figure 1), the optical axis is at a fixed tilt of $20^{\circ}$ from the zenith. Within the focal plane, movement is possible over $+-4^{\circ}$, so the declination coverage at any azimuth setting is only $8^{\circ}$, but telescope rotation can extend total declination coverage to $48^{\circ}$.

Designing the telescope this way greatly simplifies mirror supports, and much larger segments can be figured from thin blanks and supported by simple mounts than could be done for a telescope whose primary had to change orientations. We have settled on a design based on circular segments. Hexagons provide no advantage as we wish to spread the segments to increase integration time. Choosing segment size involves many considerations. Alignment would be simpler with fewer segments, so one alternative is to use large segments $(>2-\mathrm{m})$ cast as described by Angel and Hill (1982). Blank and figuring costs are expensive, however, and there is as yet no routine source for producing many such segments. The next alternative would be blanks produced by fusion of single plates onto underlying cellular arrays (Hill and Ange1 1982); the maximum size of $1.2-\mathrm{m}$ is set by the sheet Pyrex available for faceplates. This technique is promising, but also not in routine, reliable use. Blanks of 0.9-m and smaller can be cut directly from sheets of Pyrex $4.1 \mathrm{~cm}$ thick, and such blanks are marketed commercially. While production of the blanks is cheap, they are sufficiently thick that small ones can be supported by simple 3-point mounts. Scaling from the support systems described by Pearson (1980), the maximum size for 3-point support is about $0.4 \mathrm{~m}$. Yet, each segment must have 3 actuators for alignment, so actuator costs scale directly with the number of segments required, as does alignment effort. So there can be a gain in utilizing more sophisticated mounts in order to use larger but fewer segments. Because of these various considerations, opinions may differ as to the optimum choice of segment size. To us, the best compromise seems to be use of the single sheet, $0.9 \mathrm{~m}$ segments. That they are cast flat (rather than slumped or curved) is no problem, because the sagittal depth is only $4 \mathrm{~mm}$ for the radius of curvature chosen. This small sagittal depth also means the blanks closely match the idealized segments for which supports were analyzed by Pearson (1980) and Nelson et a1. (1982). These analyses show that even a 6 point support is insufficient for these blanks. Given that, we plan to float the blanks at many points upon a honeycombed aluminum cell, which is in turn supported by the 3 actuators. Remember that weight is not a major factor because the segment array does not move.

The design we illustrate utilizes a symmetrical array of such segments configured as shown, requiring 73 segments, an arrangement that approximates a 

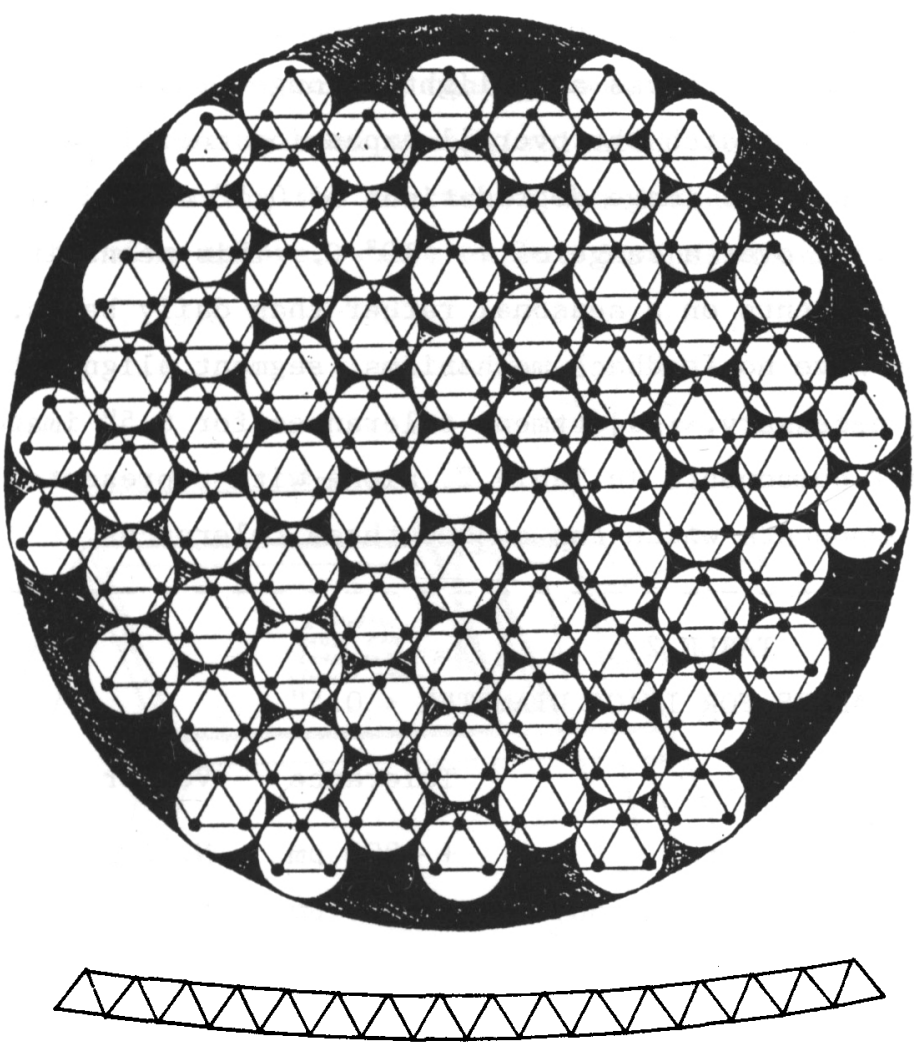

circular array. This array with actuator 10cations and geometry of the support truss is shown in Figure 2. With this configuration and a corrector observing an aperture of $8-\mathrm{m}$ diameter, the effective size of the telescope as a function of integration time is shown in Table 1 . It acts as a 7-m telescope for an integration time of 15 minutes.

Figure 2: Segment, actuator and truss configuration

TABLE 1

EFFECTIVE APERTURE OF PSSST

$\begin{aligned} \text { Integration Time } & \begin{array}{c}\text { Effective Aperture } \\ \text { (Central Dec.) }\end{array} \\ 5 \mathrm{~min} & \text { always }>7.2 \mathrm{~m} \\ 10 \mathrm{~min} & \text { always }>7.1 \mathrm{~m} \\ 15 \mathrm{~min} & \text { always }>6.9 \mathrm{~m} \\ 20 \mathrm{~min} & \text { always }>6.7 \mathrm{~m} \\ 30 \mathrm{~min} & \text { always }>6.5 \mathrm{~m} \\ 40 \mathrm{~min} & \text { always }>6.2 \mathrm{~m}\end{aligned}$

Effective Aperture
$\left(4^{\circ}\right.$ from Central Dec.
always $>6.3 \mathrm{~m}$
always $>6.3 \mathrm{~m}$
always $>6.2 \mathrm{~m}$
always $>6.1 \mathrm{~m}$
always $>6.0 \mathrm{~m}$
always $>5.7 \mathrm{~m}$

Once the gravity vector is fixed, the only source of perturbation to the primary is thermal changes. These can be minimized by designing a mirror support frame with thermal expansion properties close to those of the Pyrex mirrors. This support frame will be a space frame constructed of aluminum connectors and invar rods, with dimension ratios chosen such that the net expansion coefficient matches that of Pyrex. This can be done precisely only if all rods in the space frame are of the same length, but works because invar has an expansion coefficient less than Pyrex but aluminum is greater. Our frame 
cannot precisely accomplish this correction because the frame is slightly curved to match the array so rods in the lower truss are slightly longer than those in the upper. It should be possible to match the overall expansion coefficient with Pyrex to within $\backsim 3 \times 10^{-7}$, so that image degradations of $0.5^{\prime \prime}$ caused by temperature changes will not arise over a range of $+-10^{\circ} \mathrm{C}$. This means it will be possible to align the segments on a seasonal rather than daily basis. Consequently, mirror actuators have no feedback mechanisms; segment alignment is to be done by inspection when necessary. Adjustment tolerances for $0.5^{\prime \prime}$ image diameter are summarized for all components in Table 2, along with representative vendors who claim to supply components which can exceed these tolerances.

TABLE 2

COMPONENT TOLERANCES FOR IMAGE DIAMETER $<0.5^{\prime \prime}$

\begin{tabular}{lcc}
\multicolumn{1}{c}{ Component } & Tolerance & Vendor \\
Mirror Segments: radius of curvature & $+-900 \mu \mathrm{m}$ & N. Cole \\
Mirror Actuators: absolute setting (piston) & $+-20 \mu \mathrm{m}$ & Newport \\
Mirror Actuators: relative setting (tilt) & $+-0.4 \mu \mathrm{m}$ & Newport \\
X,Y Track and Motors: absolute setting (10") & $+-60 \mu \mathrm{m}$ & Klinger \\
X,Y Track and Motors: relative setting $\left(.1^{\prime \prime}\right)$ & $+-0.6 \mu \mathrm{m}$ & Klinger \\
Z Motor and Track: absolute setting (focus) & $+-400 \mu \mathrm{m}$ & Klinger \\
$\theta, \phi$ Gimbal: absolute setting (collimation) & $+-8^{\prime \prime}$ & Newport \\
$\theta, \phi$ Gimbal: relative setting (tracking) & $+-0.2^{\prime \prime}$ & Newport
\end{tabular}

Note that flexure errors do not have to be controlled to these tolerances; because the gravity vector is fixed, flexure corrections are contained in a single look-up table within the control computer.

Several other major cost savings are incorporated in this design. The bullding rotates with the telescope, so the only opening needed is immediately in front of the focal plane. Instrumentation (except for direct CCD imagery or a compact prime focus CCD spectrograph) will be coupled to the telescope by fiber optics. This is already done for all observing with our existing 1.6-m telescope at Black Moshannon Observatory; spectrographs are simply assembled on an optical bench, and light is brought to them. The same spectrographs constructed for the $1.6-\mathrm{m}$ telescope will be directly transferable. Restricting the telescope to special purpose observing will greatly reduce maintenance and operating costs; setting will be so accurate that observing can be done in a radio-telescope mode, whereby the astronomer need only supply co-ordinates and not be present for observing. 


\section{FOCAL PLANE SYSTEM}

A telescope such as that proposed places a11 of the demands for acquisition and tracking upon a sophisticated focal plane system. This system must be capable of moving the corrector and detector over a focal surface that is a section of a sphere, whose extent depends upon the total integration time de-

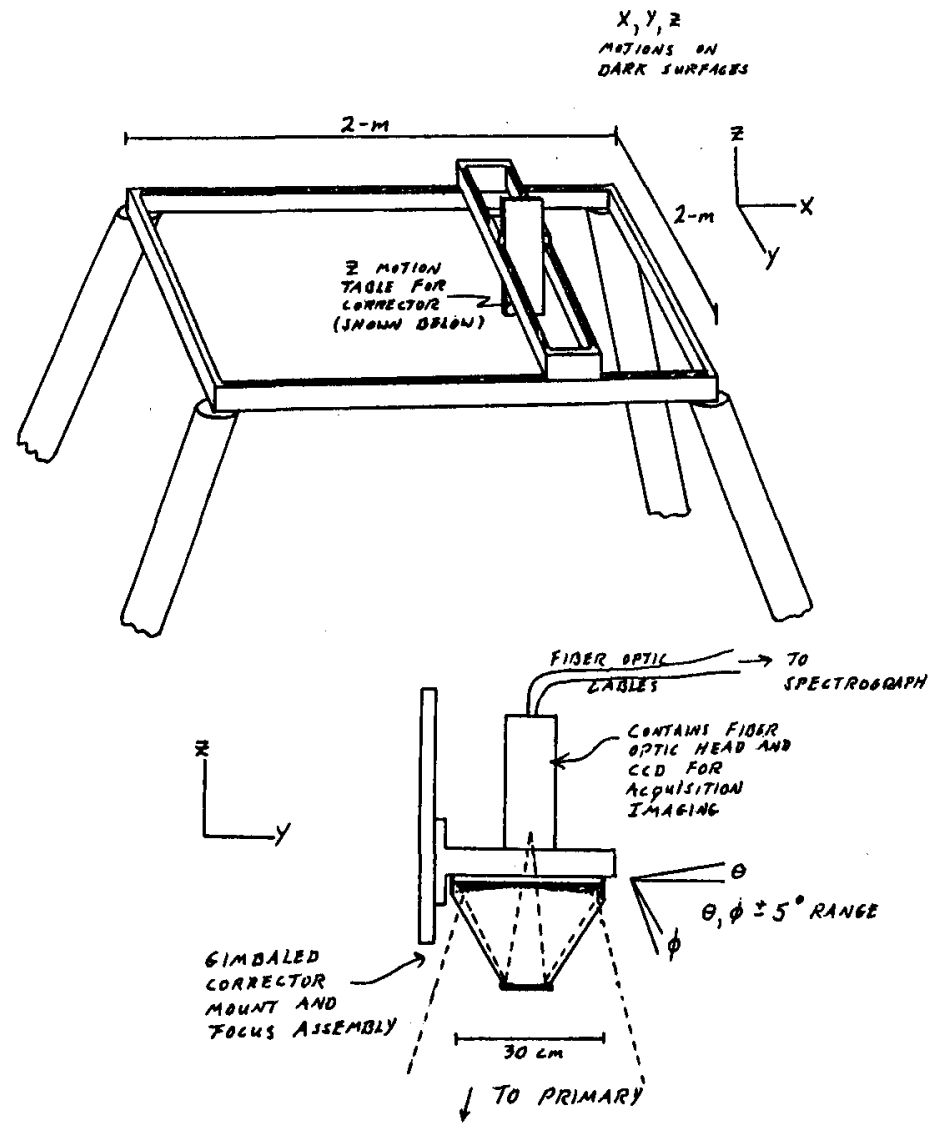

Figure 3: Focal plane system for PSSST; corrector shown to scale sired (Figure 3). Tolerances are set by the acquisition and tracking accuracy required, and by the focus and collimation requirements to achieve the desired image quality. Sources of image degradation and design of a two element corrector are discussed by Shectman

(1983). To achieve tolerances of $0.5^{\prime \prime}$ in all of these properties, mechanical tolerances for our design are approximately as shown in Table 2 . Movement of the focus in $X, Y, Z$ directions will be controlled by encoded stepping motors driving precision screws; $\theta, \phi$ motions of the gimbaled corrector mount will be

controlled over the restricted range needed by motorized micrometers. For a given setting of the telescope azimuth, the image point will move with five degrees of freedom: $\mathrm{X}, \mathrm{Y}, \mathrm{Z}$ motions to position the image point on the spherical image surface, and $\theta, \phi$ motions to position the corrector tangent to this surface. All of these co-ordinates change with time to track an object, so motions will be controlled by a super-micro computer.

In principle, "it's all software". Our in-house effort at present is to construct and control a prototype focal plane system. It is important to determine the tolerances that can actually be achieved with available hardware and to produce ready-to-run control programs. It is possible to build and gain experience with a working focal plane system without having to construct the 
full-size telescope. Various optical simulations can be imagined, and an alternative way to test accuracy and reproducibility is to use inclinometers (tiltmeters) to determine the absolute setting of the image point. Such devices are cheaply available that can measure angles to $00.1 "$ (Westphal et al. 1983). Because every position on the spherical focal surface corresponds to a unique reading on two orthogonal inclinometers, these can determine an accurate position for any point of the sky imaged by the telescope. Also, tiltmeters on one or more mirrors monitor absolute alignment of the primary array relative to the focal plane system.

Obviously, several correctors could be used simultaneously within a focal plane system to observe different objects. This is a potentially significant gain in efficiency compared to a conventional telescope. Our own experiments are to be directed only to a single image point for the time being, but it would be straightforward to install several duplicate systems, and the computer to be used has the capacity to operate several systems together.

At the moment of this Colloquium, we are only to the point of installing the hardware described in our test lab. Encoding position information, devising simulations, and coupling the inclinometers is the initial phase of effort for the focal plane system. Mirror supports and actuator control systems are also being examined. Discussions are underway with vendors who can supply and figure the mirror segments. A site has been chosen (Flagstaff, Arizona) based on our judgment of the best compromise between scientific return and cost of construction/operation. The pace of construction depends, as for most such projects, on the pace of funding. It is anticipated that testing of the focal plane system with a limited number of mirror segments will be done on the Penn State campus, but the full assembly of the telescope will take place only at the final site.

\section{SCIENTIFIC OBJECTIVES}

Our profession is not building telescopes. We are at present pursuing survey projects of emission-line galaxies and quasars together with spectroscopic monitoring projects of stars with suspected solar-like surface activity. These are being done with our 1.6-m telescope, and it has become clear that many of our scientific objectives require greater aperture. Our goal is to obtain that aperture with minimal cost in money, manpower and time. If the telescope proposed becomes available, its initial targets will be well defined. For extragalactic observations, the first years of operation will be dedicated to following up large samples of quasars and active galaxy candidates discovered primarily on objective prism surveys to 19 th magnitude. There are one to a few such objects per square degree, and it is important to understand how 
they are distributed in the universe. Not only are questions of quasar evolution important and still unanswered, but there is much to be learned about the distribution of galaxies in the local universe. There is no lack of targets; $10^{6}$ galaxies and $10^{5}$ quasars are visible to 19 th mag. in the accessible declination range. Observing decisions will still have to be made.

During bright time, this telescope will be devoted to monitoring observations of $\mathrm{H} \alpha$ and the $\mathrm{Ca}$ II infrared triplet in G-M stars. This is an extension of a project already underway both with the 1.6-m and with a 1-m telescope at Lowell Observatory, in collaboration with D. Mihalas at High Altitude Observatory. The objective is to understand magnetic cycles in stars by observing those indicators known from the sun to correlate with magnetic activity. Because such cycles are of decade lengths, long term, dedicated observations are essential. We are aware of no other existing or proposed telescopes that can undertake such dedicated synoptic observations of many stars.

It is important to recognize that realizing the feasibility of the telescope described was in large measure due to the extensive research and development already done by the many people involved in preparing for the next generation of large telescopes. In particular, details have been shaped to a great extent by ideas of S. Shectman, R. Ange1, and optician N. Cole, to whom we express much appreciation for various helpful discussions. A spectroscopic survey telescope is by no means a substitute for a very large, general purpose National facility. Even if the former is built first, it will owe its genesis to the thinking which went toward the latter.

REFERENCES

Ange1, R. and Hil1, J. 1982 in SPIE 332, Advanced Technology Optical Telescopes, ed. G. Burbidge and L. Barr (Bellingham, Wash.: SPIE) p. 212.

Hi11, J. and Ange1, R. 1982, Telescope Making, 14, 24.

Huchra, J., Davis, M., Latham, D. and Tonry, J. 1983, Astrophysical Journa1 Supp1., 52, 39 .

Mertz, L. 1980 in Optical and Infrared Telescopes for the 1990s, ed. A. Hewitt (Tucson: KPNO) p. 957.

Nelson, J.E., Lubliner, J. and Mast, T.S. 1982, in SPIE 332, p. 212.

Pearson, E. 1980 in Optical and Infrared Telescopes for the 1990s, ed. A. Hewitt (Tucson: KPNO) p. 555.

Schmidt, M. and Green, R. 1983, Astrophysical Journa1, 269, 352.

Shectman, S.A. 1983 in SPIE 444, Advanced Technology Optical Telescopes II, in press.

Vaughn, A.H. and Preston, G.W. 1980, Pub. Astron. Soc. Pac., 90, 267. 
Westphal, J.A., Carr, M.A., Miller, W.F. and Dzurisin, D. 1983, Rev. Sci. Instruments, 54, 415.

Wilson, 0.C. 1978, Astrophysica1 Journa1, 226, 379. 